\title{
Contact ability optimization of the surface of titanium parts with different stiffness during flat grinding by highly porous wheel
}

\author{
Yakov I. Soler ${ }^{1, *}$, Dinh Si Mai ${ }^{1}$, and Denis Yu. Kazimirov ${ }^{1}$ \\ ${ }^{1}$ Irkutsk Nation Research Technical University, Department of Technology and Equipment of \\ Machinery Production, 664074, Irkutsk, Russian Federation
}

\begin{abstract}
In order to improve the contact performance properties, we present optimization of flat grinding of titanium parts in accordance with two parameters: the relative bearing length of the profile and the relative bearing part of the parts' surface that allow to assess the quality of the surface at the micro- and macro-scale. It is established that in order to achieve the set goal, grinding should be carried out with a minimum lateral feed rate $s_{\text {пा }}=2.0 \mathrm{~mm} /$ double pass, and the remaining technological parameters are selected depending on the rigidity of the parts and the direction of its variation.
\end{abstract}

\section{Introduction}

The most important operational properties of machine parts, such as wear resistance, contact stiffness, etc., depend significantly on the contact interaction of their working surfaces, finally formed at the final stage of processing, including grinding. However, grinding of parts made of titanium alloys, which are widely used in aeronautics and space technology, shipbuilding, oil and chemical engineering for manufacture of critical products, is used much less often than other structural materials. The reason for this is related to the sticking of chips on the working surfaces of abrasive tools made of silicon carbide and electrocorundum. This is due to the high adhesion activity between titanium and traditional abrasives at operating cutting temperatures. A radical solution to this problem is the use, when grinding titanium alloys, of highly porous wheels made of cubic boron nitride and silicon carbide [1].

It is known that geometry of the real surface of any part is significantly different from the ideal one. This is due to the fact that after any method of processing on the surface of the real part, with which the contacting of machine parts begins, there are macro- and micro roughnesses of different sizes $[2,3]$. Among the microroughness parameters of the surface, the relative bearing length of the profile $t_{p}(p=\overline{5 ; 90})$ characterizes the actual bearing area of the machine parts at the microscale [4,5]. It mainly affects the wear resistance of movable joints, the strength of compression fits and the size of local plastic plastic deformation of surfaces when they come into contact. During operation, large surface

\footnotetext{
*Corresponding author: solera@istu.irk.ru
} 
irregularities under load are quickly deformed or abraded, which leads to a weakening of the tension or a sharp increase in the initial gap. In [6, 7], there was a study of the relationship between the probability of contact of an abrasive tool with a workpiece during grinding. The obtained results make it possible to trace the regularities in the formation of the surface microrelief parameters on the base section, taking into account possible changes and deviations of the elements of the cutting regime and the state of the working surface of the tool.

In operation, as a result of macro-irregularities, adhesion and friction interaction processes, as well as local surface destruction (wear) processes occur at contact areas of mating surfaces. The decrease in the bearing surface area of the parts leads to a sharp increase in local loads in the form of forces that tend to plastically deform them [8]. Their presence leads to the need to study the dimensions of the bearing surface area of the parts that determine the zone of probable surface contact of machine parts when they are mated. There are different approaches to determining this parameter, such as adhesion, geometric, electrical, etc. [9-11]. These methods for estimating the bearing area have not found wide application because of the complexity of its determination and the high cost of the instruments used. To solve this problem, a technique called digital topography was developed, in which a new indicator was proposed - the relative bearing part $t_{f M}$ [12]. The study of the parameter $t_{p}$ together with the relative bearing area $t_{f M}$ of the parts' surface allows for a more complete understanding of their contact ability.

In modern production, optimization is one of the main directions for improving the process of machining. In this case, the criterion is usually used for the cost of production or the productivity of the process. At the finish operations, optimization is also associated with improving product quality [13-15].

In connection with the foregoing, this research is devoted to optimizing the parameters of the relative bearing part and the relative bearing length of the surface profile in the case of flat grinding of titanium parts of various rigidity by a high-porous wheel of cubic boron nitride using the simplex-planning method.

\section{Experiment procedure}

Full-scale experiments were carried out on a 3E711B surface grinding machine with a highly porous wheel CBN30 B107 100 OV K27 КФ40. The object of the study was samples from a high-strength titanium alloy BT22 (GOST 19807-91) with dimensions $\mathrm{L} \times \mathrm{B} \times \mathrm{H}=40 \times 40 \times 50 \mathrm{~mm}$, polished along the $\mathrm{L} \times \mathrm{B}$ plane. To model the stiffness of the parts, a frame structure was used, which, due to the change in the height of the ribs and their bolting at different heights from the installation base, made it possible to vary its compliance [16]. The latter was used on the workpiece, which retained unchanged size and shape. Three levels of rigidity $j$ were used in the study: $j \in[380 ; 4174] \mathrm{N} / \mathrm{mm}$ - not rigid; $j$ $\in[4174 ; 7426] \mathrm{N} / \mathrm{mm}$ - medium-rigid; $j \in[7426 ; 11220] \mathrm{N} / \mathrm{mm}$ - highly rigid. The last subgroup received such a name conditionally, as it has a greater compliance in comparison with absolutely rigid. Due to the rotation of the device, the compliance of the parts in two mutually orthogonal directions $(w=1 ; 2)$ coinciding with the supply vectors was provided: 1 - in the transverse direction $\left(s_{\Pi}\right), 2$ - in the longitudinal direction $\left(s_{\text {пр }}\right)$. The parts of the plate type were conventionally considered absolutely rigid $(\mathrm{j} \rightarrow \infty)$ when they were fixed by stuck to a plate placed directly on the machine table.

It is known that process modeling is the first necessary step to optimize its parameters. For this purpose grinding was carried out by varying the input factors according to the Doptimal plan. In this case, the variation ranges of technological parameters are the following: the wheel speed $v_{\mathrm{K}}=29 \mathrm{~m} / \mathrm{s}$, the longitudinal feed rate $s_{\text {пр }}=5-18 \mathrm{~m} / \mathrm{min}$, the 
transverse feed $s_{\text {п }}=2-10 \mathrm{~mm} / \mathrm{d}$. stroke, the cutting depth $t=0.005-0.02 \mathrm{~mm}$, operating allowance $z=0.1-0.3 \mathrm{~mm}[16]$. In the models, there is an operating allowance $z$ and workpiece rigidity $j$, which are usually not taken into account in the regulations for the cutting modes.

The relative bearing part $t_{f M}$ of the surface is determined by the procedure [12]. The relative bearing profile length tp at different levels of the section $p=5 ; 25 ; 50 ; 70 \%$ is measured with a profilograph-profilometer model 252 of "Caliber" factory in two directions $\mathrm{r}=1 ; 2$, located parallel to the vectors $s_{\text {п }}$ and $s_{\text {пр }}$. This is due to the formation of directional roughness on the parts' surface during pendulum grinding.

Under the conditions of the optimization, to compare the obtained performance regimes, the main (machine) time $T_{0}$ is calculated from the expression:

$$
T_{0}=\frac{l_{m} B_{m}}{1000 s_{m p}\left(s_{m} / 2\right)}\left(\begin{array}{l}
z \\
t
\end{array}+i\right), \min
$$

where $l_{\mathrm{m}}=\left(l+l_{\mathrm{sp}}+l_{\text {mep }}\right)-$ grinding length, $\mathrm{mm} ; l$ - ground surface length, $\mathrm{mm} ; l_{\mathrm{sp}}-$ plunging length, mm; $l_{\text {rep }}-$ length of overrun, $\mathrm{mm} ; B_{\text {mI }}=B+2 T+10-$ grinding width, $\mathrm{mm} ; I_{-}$wheel height, $\mathrm{mm} ; i$ - number of spark-out moves. In this case $Z_{\mathbb{I}}=40+30=70$ $\mathrm{mm} ; B_{\operatorname{mI}}=40+2 \times 20+10=90 \mathrm{~mm} ; \tilde{i}=0$ (operation without spark-out at the end of the cycle).

\section{Main results and their discussion}

As a result of modeling, regression models for all the studied surface quality parameters were obtained. As an example, models for the transverse stiffness $j_{1}$ in the transverse direction $(\mathrm{r}=1)$ of the measurement $t_{p}$ are given:

$$
\begin{gathered}
\hat{t}_{M(1)}=\left(8.86-0.03 s_{\text {mp }}-0.05 s_{\text {II }}-99.57 t+3.49 z+5.06 \times 10^{-4} j_{1}-\right. \\
\left.-6.92 \times 10^{-6} s_{\text {mp }} j_{1}-1.30 s_{\text {mI }} z-4.36 \times 10^{-8} j_{1}{ }^{2}\right)^{2}, \% \\
t_{50 \text { (11) }}=52.72-0.78 s_{\text {mp }}-147.03 t+19.71 z-2.38 \times 10^{-4} j_{1}+23.09 s_{\text {mp }} t-1864.43 t z, \% ;
\end{gathered}
$$

From (2) it is revealed that parameters $\hat{t}_{\mathcal{M}(1)}$ are influenced in different ways by all technological parameters, including the operational allowance $z$ and the rigidity of the grinded parts $j_{1}$. However, according to (3) only some of them influence the other parameters.

The figure 1 shows the response surfaces $t_{\mathcal{M} 1}$, depending on the variation of transverse feed and stiffness. It was found that in both cases $(z=0.1$ and $0.3 \mathrm{~mm})$ with an increase in lateral feed from 2.0 to $10 \mathrm{~mm}$ /double pass the parameter $t_{M(1)}$ decreases, especially with an increased allowance (Fig. 1b). In addition, it was found that the highest value $t_{\Omega M(1)}$ was predicted at a medium rigidity of the grinded parts $j_{1}=5800 \mathrm{~N} / \mathrm{mm}$. 


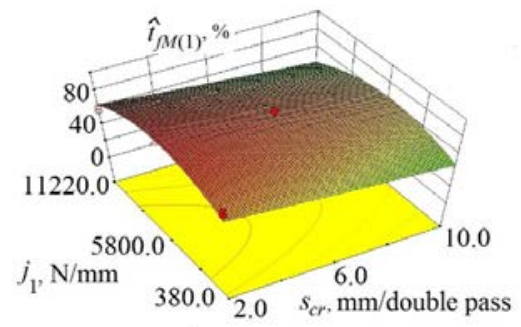

a)

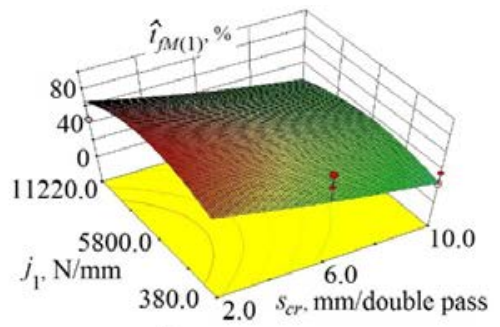

b)

Fig. 1. Effect of transverse feed $s_{\pi}$ and stiffness $j_{1}$ of parts on the response surface $\hat{t}_{\mathcal{M}(1)}$ when $s_{\text {mp }}=5 \mathrm{~m} / \mathrm{min}$., $t=0.005$ micrometre: $\mathrm{a}-\mathrm{z}=0.1 \mathrm{~mm} ; \mathrm{b}-0.3 \mathrm{~mm}$

It is revealed that the parameters studied were the largest for different values of technological factors, which makes it difficult to select the operating mode to ensure an increase in the complex of parameters under study that ensure the contact property of the parts' surface. To solve this problem, multiparametric optimization was performed by this method [16]. At the same time for parameter $t_{f M}$ ranks $\mathrm{R}=5$ are assigned, the exponent of the scale function is $n=[10 ; 1]$; for the rest - by default of the program: $R=3, n=[1 ; 1]$. The optimization efficiency is characterized by the desirability $d \in[0 ; 1]$ and minimum function (1).

Table 1. Results of optimizing the bearing capacity for grinding of absolutely rigid parts

\begin{tabular}{|c|c|c|c|}
\hline $\begin{array}{l}\text { Technological } \\
\text { factors }\end{array}$ & Surface quality parameters, $\%$ & $d$ & $\begin{array}{l}T_{0} \\
\min \end{array}$ \\
\hline $\begin{array}{c}s_{\mathrm{mp}}=8.79 \mathrm{~m} / \mathrm{min} ; \\
s_{\mathrm{mI}}=2.00 \mathrm{~mm} / \text { double } \\
\text { pass; } \\
t=0.02 \mathrm{~mm} ; \\
z=0.10 \mathrm{~mm}\end{array}$ & $\begin{array}{l}\hat{t}_{5(1)}=3.44 ; \hat{t}_{25(1)}=21.30 ; \hat{t}_{50(1)}=58.96 ; \\
\hat{t}_{10(1)}=80.09 ; \hat{t}_{5(1)}=2.05 ; \hat{t}_{25(2)}=11.69 ; \\
\hat{t}_{50(2)}=41.00 ; \hat{t}_{10(2)}=73.68 ; \hat{t}_{\mathcal{M}}=61.36\end{array}$ & 0.54 & 3.58 \\
\hline
\end{tabular}

First, let's consider the optimization of these parameters when grinding absolutely rigid parts from BT22. For this purpose, the objective functions are assigned: parameters $t_{F}, \mathrm{p}=$ $5,25,50,75 \%$ and $t_{\mathcal{M}}$ - maximization to increase the contact capacity of parts under the constraints: $s_{\mathrm{mp}}, s_{\mathrm{m}}, t, z, j$ - in the range from minimum to maximum. As shown in Table 1 , the obtained grinding result allows to obtain the relative surface bearing part $\hat{t}_{\mathcal{M}}=61.36 \%$, which turned out to be more than 2 times as compared with the result $\left(\hat{t}_{\Omega M}=44.91 \%\right)$ predicted in the optimization taking into account the process productivity at the final stage [16]. However, to ensure this quality, the main time increases by (1) in $3.58 / 0.53=6.75$ times due to the decrease in longitudinal and transverse feeds. In addition, it was revealed that the parameters $\hat{t}_{y}$ at different levels $(\mathrm{p}=5,25,50,70 \%)$ depend significantly on the direction of their measurement (directed topography): they turned out to be much larger in the transverse direction $r=1$.

Similarly, the optimization of the bearing capacity of the parts' surface for nonrigid parts was carried out. It is established that the quality parameters under study vary mainly depending on the compliance of the grinded parts and the direction of its variation: $w=1 ; 2$ (Table 2). Thus, for nonrigid parts the parameter $\hat{t}_{\mathcal{M}(2)}=81.02 \%$ and turned out to be $9 \%$ more compared to $\hat{t}_{\mathcal{M}(1)}$ in the transverse direction; for medium-rigid parts $-t_{\mathcal{M}(1)}=90 \%$ 
in relation to $\hat{t}_{\mathcal{M ( 2 )}}=73.92 \%$; for highly rigid parts $-\hat{t}_{\mathcal{M}(\mathrm{w})}$ predicted to be the same for $w$ $=1 ; 2$. The property of directed topography of the surface was preserved. However, in these cases, the relative bearing lengths of the profile measured in the longitudinal direction at different section levels $(\mathrm{p}=5,25,50,70 \%)$ turned out to be larger than their transverse counterparts. In addition, it was found that grinding of nonrigid parts provides an increase in the relative bearing part of the parts' surface compared to absolutely rigid parts (Table 1). However, for this purpose, grinding must be carried out with minimum longitudinal and transverse feeds, which reduces the productivity of the process up to 10 times.

Table 2. Results of optimization of the bearing area and its scattering during grinding of nonrigid parts

\begin{tabular}{|c|c|c|c|c|}
\hline$w$ & $\begin{array}{l}\text { Technological } \\
\text { factors }\end{array}$ & Surface quality parameters, $\%$ & $d$ & $\begin{array}{l}T_{0} \\
\text { min }\end{array}$ \\
\hline \multicolumn{5}{|c|}{ For nonrigid parts $\left(j_{r}=380 \mathrm{H} / \mathrm{mm}\right)$} \\
\hline 1 & 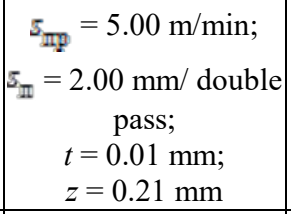 & $\begin{array}{c}\hat{t}_{5(11)}=1.91 ; \hat{t}_{15(11)}=13.73 ; \hat{t}_{50(11)}=50.72 ; \\
\hat{t}_{30(11)}=79.02 ; \hat{t}_{5(21)}=2.64 ; \hat{t}_{25(21)}=21.10 ; \hat{t}_{50(21)}= \\
61.63 ; \hat{t}_{10(21)}=85.35 ; \hat{t}_{M(1)}=72.00\end{array}$ & 0.54 & 26.46 \\
\hline 2 & $\begin{array}{c}s_{\mathrm{mp}}=5.00 \mathrm{~m} / \mathrm{min} \\
s_{\mathrm{m}}=2.00 \mathrm{~mm} / \text { double } \\
\text { pass; } \\
t=0.01 \mathrm{~mm} \\
z=0.30 \mathrm{~mm}\end{array}$ & $\begin{array}{c}\hat{t}_{5(12)}=2.28 ; \hat{t}_{15(12)}=13.77 ; \hat{t}_{50(12)}=48.34 ; \\
\hat{t}_{30(12)}=79.02 ; \hat{t}_{5(22)}=2.66 ; \hat{t}_{25(22)}=19.07 ; \hat{t}_{50(22)}= \\
56.11 ; \hat{t}_{10(22)}=81.78 ; \hat{t}_{M(2)}=81.02\end{array}$ & 0.57 & 37.80 \\
\hline \multicolumn{5}{|c|}{ For highly rigid parts $\left(j_{r}=11220 \mathrm{H} / \mathrm{mm}\right)$} \\
\hline 1 & \begin{tabular}{|c|}
$s_{\mathrm{mp}}=5.00 \mathrm{~m} / \mathrm{min}$ \\
$s_{\mathrm{m}}=2.00 \mathrm{~mm} /$ double \\
pass; \\
$t=0.01 \mathrm{~mm}$ \\
$z=0.10 \mathrm{~mm}$
\end{tabular} & $\begin{array}{c}\hat{t}_{5(11)}=1.65 ; \hat{t}_{15(11)}=13.10 ; \hat{t}_{50(11)}=47.04 ; \\
\hat{t}_{70(11)}=77.23 ; \hat{t}_{5(21)}=2.64 ; \hat{t}_{15(21)}=21.10 ; \\
\hat{t}_{50(21)}=61.63 ; \hat{t}_{70(21)}=85.35 ; \hat{t}_{M M(11)}=64.35\end{array}$ & 0.49 & 12.60 \\
\hline 2 & \begin{tabular}{|c|}
$s_{\mathrm{mp}}=5.00 \mathrm{~m} / \mathrm{min}$ \\
$s_{\mathrm{m}}=2.00 \mathrm{~mm} /$ double \\
pass; \\
$t=0.01 \mathrm{~mm}$ \\
$z=0.30 \mathrm{~mm}$
\end{tabular} & $\begin{array}{l}\hat{t}_{5(12)}=1.65 ; t_{25(12)}=13.77 ; \hat{t}_{50(12)}=48.34 ; \\
t_{70(12)}=79.02 ; \hat{t}_{5(22)}=2.66 ; t_{25(22)}=22.25 ; \\
t_{50(22)}=63.22 ; \hat{t}_{10(22)}=86.52 ; \hat{t}_{\Omega M(22)}=66.82\end{array}$ & 0.55 & 37.80 \\
\hline
\end{tabular}

Table 2 shows that the operational allowance is an important factor affecting the quality of the treated surface and the productivity of the process. Its choice is made depending on the rigidity of the parts and the direction of its variation: with the transverse compliance of the parts $(w=1)-z=0.21 \mathrm{~mm}$ for nonrigid parts, $z=0.10 \mathrm{~mm}$ for medium and highly rigid parts; at longitudinal compliance of parts $(w=2)-z=0.30 \mathrm{~mm}$. In connection with the said grinding of the parts of the transverse compliance, the main transition time is reduced to 3 times compared with its analog in the orthogonal direction.

\section{Conclusions}

1. The method of flat grinding optimization by the contact ability of parts was implemented, which is important for their increased requirements for wear resistance, contact stiffness, tightness, etc. 
2. The effect of the operating allowance on the formation of the surface quality during grinding of titanium parts was confirmed.

3 . To increase the relative bearing surface $t_{\mathcal{M}}$ of the surface, grinding is recommended to be carried out with a minimum lateral feed $s_{\mathrm{m}}=2 \mathrm{~mm} /$ double pass. The remaining parameters are selected depending on the rigidity of parts and the direction of its variation. In this case, the main processing time is increased several times in comparison with the optimization results when productivity is taken into account.

\section{References}

1. V.A. Nosenko, S.V. Nosenko, Technology of Metal Grinding: monograph (Staryy Oskol: TNT, 2012)

2. N.B. Demkin, E.V. Ryzhov, Surface Quality and Contact of Machine Parts (M.: Mashinostroenie, 1981)

3. A.G. Suslov, V.F. Beyazychny, Yu.V. Panfilov, S.G. Bishutin, I.V. Govorov, A.O. Gorlenko, V.P. Tikhomirov, Engineering of the Parts' Surface (M.: Mashinostroenie, 2008. - $318 \mathrm{p}$.

4. Popov V.M. Heat Transfer in the Contact Zone of Detachable and One-piece Joints (M.: Energia, 1971)

5. O.A. Leonov, Yu.G. Vergazova, Innovative Science, 1 (2016)

6. S. Bratan, S. Roshchupkin, D. Revenko, Probabilistic Approach for Modeling Electroerosion Removal of Grinding Wheel Bond, Procedia Engineering V. 206, 14261431 (2017)

7. S. Bratan, A. Kolesov, S. Roshchupkin, T. Stadnik, Theoretical-probabilistic model of the rotary belt grinding process, MATEC Web of Conferences, V. 129, 01079 (2017)

8. V.M. Khokhlov, Increase and Decrease in the Actual Contact Areas (2nd ed., Bryansk, VIMAHO, 2004)

9. V.M. Gryazev, Izvestiya of the Tula State University. Engineering Sciences, 10 (2013)

10. Yu.S. Stepanov, E.A. Belkin, V.N. Poyarkov, Fundamental and Applied Problems of Engineering and Technology, 4 (300) (2013)

11. Firsov A.M., Belyaev V.N. Patent RU 2301967 C1, 2006.

12. Ya.I. Soler, D.S. Mai, D.Yu. Kazimirov, ARPN Journal of Engineering and Applied Sciences, 11(17) (2016)

13. RDMU 109-77, Method of Selection and Optimization of Controlled Parameters of Technological Processes, Introduction. 19.12.1977, M.: Publishing Standards, 1978.

14. S. Kurra, N.H. Rahman, S.P. Regalla, A.K. Gupta, Journal of Materials Research and Technology, 4(3) (2015)

15. T.W. Liao, L.J. Chen, Int J Mach Tools Manuf, 34(7) (1994)

16. Ya.I. Soler, D.Sh. May, Vestnik IrSTU, 12(21) (2017) 